\title{
Real-time implementation with FPGA-based DAQ system of a probabilistic disruption predictor from scratch
}

\author{
Vega, J.
}

2018

Vega , J , JET Contributors \& Ahlgren , T 2018 , ' Real-time implementation with FPGA-based DAQ system of a probabilistic disruption predictor from scratch ' , Fusion Engineering and Design , vol. 129 , pp. 179-182 . https://doi.org/10.1016/j.fusengdes.2018.02.071

http://hdl.handle.net/10138/326690

https://doi.org/10.1016/j.fusengdes.2018.02.071

cc_by_nc_nd

submittedVersion

Downloaded from Helda, University of Helsinki institutional repository.

This is an electronic reprint of the original article.

This reprint may differ from the original in pagination and typographic detail.

Please cite the original version. 


\title{
Development of a new compact gamma-ray spectrometer optimised for runaway electron measurements
}

\author{
A. Dal Molin ${ }^{1}$ L. Martinelli, ${ }^{1}$ M. Nocente,${ }^{1,2}$ D. Rigamonti, ${ }^{2}$ A. Abba,${ }^{3}$ L. Giacomelli, ${ }^{2}$ G. Gorini, ${ }^{1,2}$ \\ A. Lvovskiy, ${ }^{4}$ A. Muraro, ${ }^{2}$ and M. Tardocchi. ${ }^{2}$ \\ ${ }^{1}$ Dipartimento di Fisica “G. Occhialini”, Università degli Studi di Milano-Bicocca, Milano, Italy \\ ${ }^{2}$ Istituto di Fisica del Plasma "P. Caldirola”, CNR, Milano, Italy \\ ${ }^{3}$ Nuclear Instruments, Lambrugo, Italy \\ ${ }^{4}$ Oak Ridge Associated Universities, Oak Ridge, TN, USA
}

(Presented XXXXX; received XXXXX; accepted XXXXX; published online XXXXX)

(Dates appearing here are provided by the Editorial Office)

A new compact gamma-ray spectrometer was developed in order to optimise the measurement of bremsstrahlung radiation emitted from runaway electrons in the $\mathrm{MeV}$ range. The detector is based on a LYSO:Ce scintillator coupled to silicon photomultipliers and is insensitive to magnetic fields. A dedicated electronic board was developed to optimise the signal readout as well as for on-line control of the device. The detector combines a dynamic range up to $10 \mathrm{MeV}$ with moderate energy non linearity, counting rate capabilities up to a few $\mathrm{MHz}$ and an energy resolution that extrapolates to a few \% in the $\mathrm{MeV}$ range, thus meeting the requirements for its application to runaway electron studies by bremsstrahlung measurements in the gamma-ray energy range.

\section{INTRODUCTION}

The ITER project is one of the most ambitious international endeavor of this century. It aims to demonstrate the production of fusion power with a ten-fold gain. ITER will also try to prove that high performance plasma operation can be reliably and safely achieved. A major obstacle in this effort could be represented by the damage caused to the first wall materials by uncontrolled runaway electron beams. As a result the study and characterization of the runaway electron population during plasma discharges has become one of the most prominent topic of the fusion research field ${ }^{1,2,3}$.

Gamma-ray spectroscopy, which is among the primary tools to investigate fast ion physics ${ }^{4,5,6,7,8}$ has also been more recently applied to studies of runaway electrons in high temperature plasmas ${ }^{3,9,10,11}$, also in view of ITER 12. Gamma-rays are born from bremsstrahlung emission when the runaway electron population interacts either with the plasma bulk or with the tokamak plasma facing components. By measuring this emission it is possible to deduce information about the runaway population.

This type of measurements poses several requirements and restrictions to the detector. First of all, the spectrometer must have a high counting rate capability to withstand the intense photon flux of runaway electron bremsstrahlung emission. Than the spectrometer has to be able to detect gamma-rays for a wide range of energies, up to several MeV. Moreover the intense magnetic fields of a tokamak forbid the use of traditional photodetectors such as photomultiplier tubes. Finally, the detector has to be compact. This is necessary for it to be implemented in the detector array configuration that is required for the tomographic reconstruction of the runaway electron spatial distribution.

The requirements of runaway electron measurements by their bremsstrahlung emission demand the development of specific gamma-ray spectrometer optimised for such purpose, which is discussed in this paper.

\section{THE DETECTOR}

The detector is based on a cerium doped lutetiumyttrium oxyorthosilicate (LYSO:Ce) scintillator crystal coupled with a silicon photomultiplier (SiPM).

LYSO is commonly used in both high energy physics and nuclear medicine applications ${ }^{13}$. Its properties can be also useful for measuring fusion plasma gamma-ray emission. In particular, the material shows high stopping power due to its high density, good light yield and high detection efficiency in the gamma-ray range. Moreover its

${ }^{\text {a)}}$ Published as part of the Proceedings of the 22nd Topical Conference on High-Temperature Plasma Diagnostics (HTPD 2018) in San Diego, California, USA.

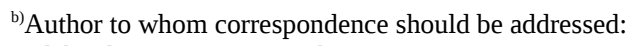
a.dalmolin@campus.unimib.it 
fast scintillation decay time (approximately $36 \mathrm{ns)} \mathrm{makes}$ this scintillator suitable for high fluxes scenarios where high counting rate capability is necessary. Due to the presence of ${ }^{176} \mathrm{Lu}$, a beta emitter, LYSO crystals display a characteristic intrinsic background radioactivity at a typical counting rate of $39 \mathrm{cps} / \mathrm{g}$. The energy range of these events covers an interval between $88 \mathrm{keV}$ and 1.2 $\mathrm{MeV}$. This intrinsic background does not interfere with the measurement of high rate events but can be used in principle to verify the detector stability between two plasma discharges. Finally, LYSO crystals are radiation hard and non hygroscopic, making them practical to handle.

Silicon photo-multipliers ${ }^{14}$ are solid state photodetectors made up by arrays of avalanche photodiodes (APDs) operating in Geiger mode. This relative novel type of light detector offers several advantages with respect to the more standard Photomultiplier tube (PMT). First of all, the gain of a SiPM detector is comparable to PMT typical values with the advantage of being insensitive to magnetic fields. This feature is of extreme importance for detectors positioned close to the tokamak intense magnetic fields. Other positive characteristics of these devices are their compactness, their relative low bias voltage operation (approximately $55 \mathrm{~V}$ ) and their robustness to radiation damage ${ }^{15}$. Despite the continuous progress made in this technology, SiPMs still show gain shifts due to temperature changes and limited energy linearity due to their finite number of APDs. Fortunately, both limitations can be corrected for with dedicated procedures.

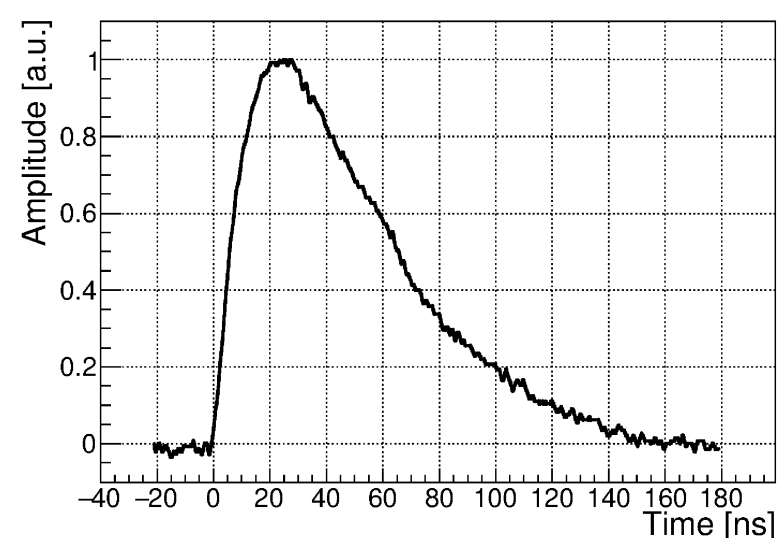

Fig. 1. Output signal of the LYSO crystal coupled with the SiPM and the dedicated readout electronics.

Finally a dedicated electronic readout circuit was developed for this detector. The original design, proposed in ${ }^{14}$ and subsequently improved in ${ }^{16,17}$, was implemented to achieve fast signals (see figure 1) in order to reach high counting rate capability and minimize pile-up events. The electronic board was further improved by adding active components to obtain desirable features such as signal amplification and an on-line correction of gain shifts caused by temperature fluctuations.

The characterization of this detector was aimed to asses three major aspects: the energy resolution, the counting rate capability and the non linearity of the device.

\section{ENERGY RESOLUTION}

Energy resolution was measured using two radioactive sources, namely ${ }^{60} \mathrm{Co}\left(\mathrm{E}_{\mathrm{\gamma}}=1173 \mathrm{keV}\right.$ and 1332 $\mathrm{keV})$ and ${ }^{137} \mathrm{Cs}\left(\mathrm{E}_{\mathrm{\gamma}}=661.7 \mathrm{keV}\right)$ for two detectors: a LYSO crystal coupled with a PMT and an identical LYSO crystal coupled with the SiPM and the dedicated readout electronics. The purpose of this procedure is to compare the performance of the proposed detector against a more traditional acquisition chain. The results of these measurements are summarized in figure 2 .
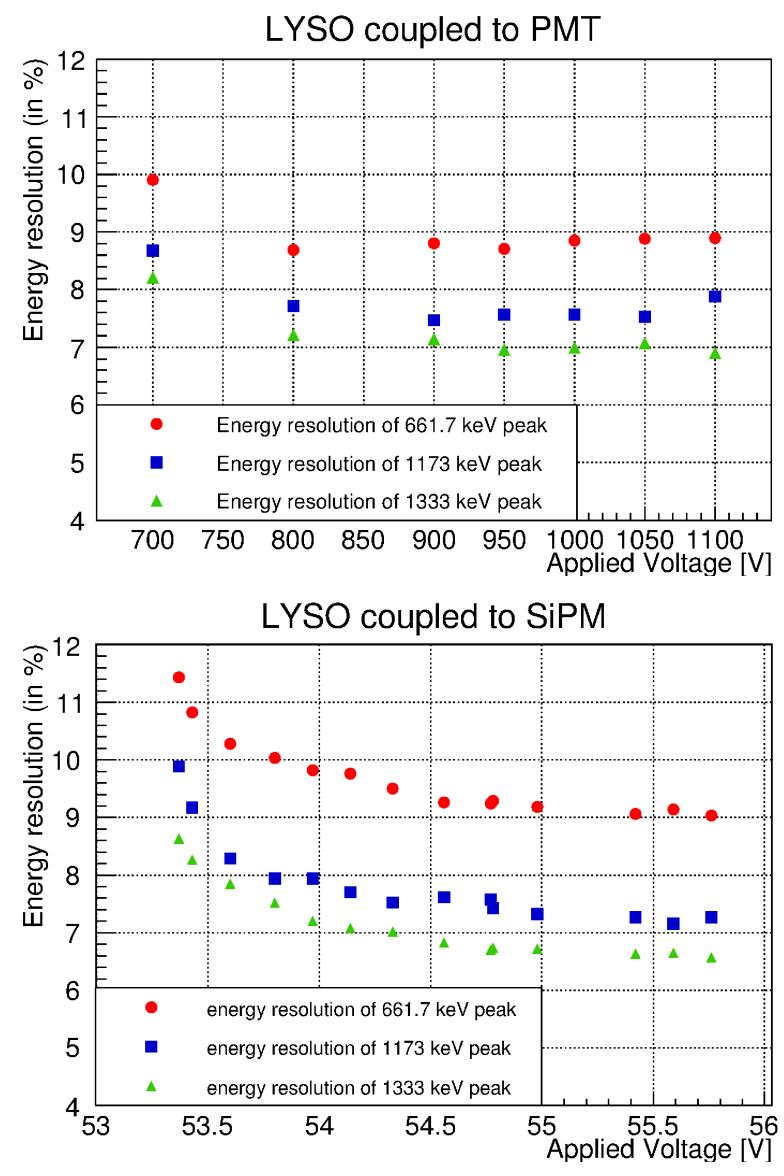

Fig. 2. Energy resolution of a LYSO crystal coupled to a PMT (top) and to a SiPM (bottom). Error bars are smaller than the size of the symbols.

For both detectors there is an optimal operation voltage at which the energy resolution is maximized. When the optimum is reached, the energy resolution is 
comparable and has a value of about $9 \%$ at $661.7 \mathrm{keV}$, as quoted by the crystal manufacturer. Hence, with the SiPM coupling we can obtain the same energy resolution results of a traditional PMT based acquisition chain, with the additional advantages of having a lower operating voltage and a more compact device insensitive to magnetic fields.

\section{MHZ COUNTING RATE CAPABILITY}

At high counting rates the signal current generated by the large amount of the SiPM ADP cells that are activated by gamma-ray detection can become significant. This relative large current generates a drop in the SiPM over voltage and a consequent reduction of the detector gain, which produces a shift of the spectrum towards lower energies ${ }^{17}$.

A similar setup to the one described in ${ }^{18}$ was used to measure the effect of these currents on the detector gain. The experimental setup consisted of two blue LEDs that can be fired independently from each other using a pulser. One LED was set to mock the light emission of the scintillation produced by a $3 \mathrm{MeV}$ photon absorbed by the LYSO crystal. This LED was fired at a constant rate of 10 $\mathrm{kHz}$ and used as a reference signal. The other LED was set to mock the light emission produced by a lower energy photon interacting with the LYSO crystal. This LED was fired at different rates scanning an interval up to $1.25 \mathrm{MHz}$ and used as perturbation. The SiPM was illuminated with the light coming from both of the LEDs using optical fibers.

In a typical measurement, data were collected over three time windows of approximately 6 second each. In the first window only the reference LED is on. In the second time window the perturbation LED is switched on at a set constant rate, causing an increase in the SiPM signal current and the shift of the reference LED energy peak in the measured spectrum due to the gain drop. In the last time window the perturbation LED is switched off and the reference LED energy peak returns to its original position in the spectrum. These measurements were repeated using two different gamma-ray equivalent energies for the perturbation LED, namely $600 \mathrm{keV}$ and 1.1 MeV. The results of these measurements are shown in figure 3 .

The relative shift of the reference peak increases in a non linear way with the rate of the perturbation. Moreover the shift tends to be more intense if the SiPM applied voltage is increased. By almost doubling the equivalent energy of the perturbation signals the relative shifts of the reference peak increases accordingly, thus confirming that this shift are indeed caused by the output currents. For a perturbation of approximately $600 \mathrm{keV}$ and a rate of $500 \mathrm{kHz}$ the shift of the reference peak is below $1.5 \%$ showing a better performance than a previous detector based on coupling a SiPM with a LaBr3 crystal ${ }^{16 \text {, }}$ 17 .
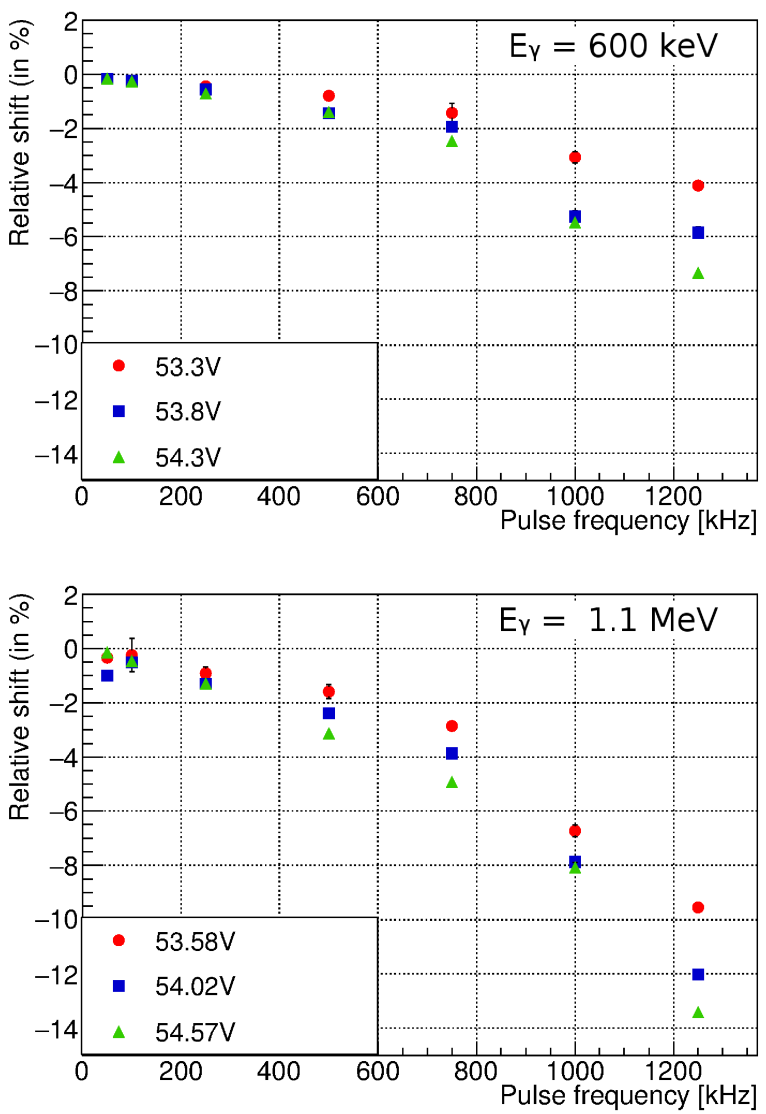

Fig. 3. Relative shift in the reference peak position due to high rate perturbation events. The left figure shows the shift caused by LED events at an equivalent gamma-ray energy of $600 \mathrm{keV}$. The shift caused by 1.1 $\mathrm{MeV}$ equivalent events is shown in the right figure. Different markers represent the different applied voltages.

\section{NON LINEARITY}

As the number of scintillation photons impinging on the SiPM approaches the total number of APD cells contained in the device, the probability that two individual photons interact with the same active pixel can be non negligible. As a consequence, the second photon is not detected by the instrument, resulting in a non proportionality of the output signal amplitude to the energy deposited in the crystal by the gamma-rays. This effect is the main cause of the photodetector non linearity.

A dedicated experimental setup was created to measure this effect. The operating parameters of a blue LED were set to mock up the scintillation of a LYSO crystal. The amplitude of the LED emission was changed to simulate different equivalent gamma-ray energies. The light emitted by the LED was split in two branches using 
WPJET4-CPR(18) 20448

A Dal Molin et al.

\section{Development of a new compact gamma-ray spectrometer optimised for runaway electron measurements.}

Preprint of Paper to be submitted for publication in Proceeding of 18th Topical Conference on High Temperature Plasma Diagnostics

(HTPD)

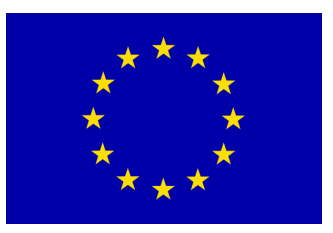

This work has been carried out within the framework of the EUROfusion Consortium and has received funding from the Euratom research and training programme 2014-2018 under grant agreement No 633053. The views and opinions expressed herein do not necessarily reflect those of the European Commission. 
This document is intended for publication in the open literature. It is made available on the clear understanding that it may not be further circulated and extracts or references may not be published prior to publication of the original when applicable, or without the consent of the Publications Officer, EUROfusion Programme Management Unit, Culham Science Centre, Abingdon, Oxon, OX14 3DB, UK or e-mail Publications.Officer@euro-fusion.org

Enquiries about Copyright and reproduction should be addressed to the Publications Officer, EUROfusion Programme Management Unit, Culham Science Centre, Abingdon, Oxon, OX14 3DB, UK or e-mail Publications.Officer@euro-fusion.org

The contents of this preprint and all other EUROfusion Preprints, Reports and Conference Papers are available to view online free at http://www.euro-fusionscipub.org. This site has full search facilities and e-mail alert options. In the JET specific papers the diagrams contained within the PDFs on this site are hyperlinked 
optical fibers. One branch illuminated the LYSO crystal coupled with the SiPM while the other carried light to an identical LYSO crystal coupled with a PMT. ${ }^{60} \mathrm{Co}$ and ${ }^{137} \mathrm{Cs}$ sources were used to calibrate the energy spectrum measured by the LYSO + SiPM detector, while ${ }^{22} \mathrm{Na}\left(\mathrm{E}_{\mathrm{y}}=\right.$ $511.0 \mathrm{keV}$ and $1274.5 \mathrm{keV}$ ) was used to calibrate the LYSO + PMT spectrum. The LED emission amplitude was changed to mock up gamma-ray at different energies within the interval of the PMT linear response. The SiPM non linearity was computed by confronting the energies measured by the two different detectors after correcting the spectra for the optical factors introduced by the two different fibers. The results are shown in figure 4 .

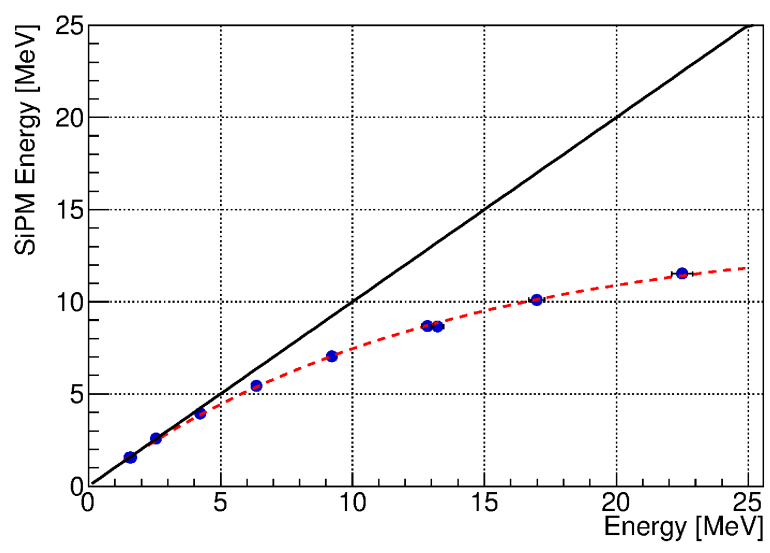

Fig. 4. Illustration of the non linearity of the SiPM. The experimental points are represented by dots and are determined by measuring the peak position from an LED source at known equivalent energy with a LYSO coupled to a PMT and to a SiPM. The solid line indicates the expected results for a perfectly linear system. The dashed line is a fit of the experimental points.

As expected the detector behavior is linear at low energies. The detector saturates above $25 \mathrm{MeV}$ for $1.5 \mathrm{~V}$ of over voltage. At $10 \mathrm{MeV}$ the non linearity of the detector is approximately $20 \%$.

\section{CONCLUSIONS}

A compact gamma-ray spectrometer based on LYSO scintillator crystal coupled with a SiPM has been developed. Dedicated readout electronics has been implemented to obtain fast signals and other desirable feature such as signal amplification and on-line temperature shift correction. Laboratory measurements showed good energy resolution of approximately $9 \%$ at
$661.7 \mathrm{keV}$. This energy resolution is sufficient to measure the continuous spectrum of runaway electron bremsstrahlung emission in fusion plasmas. The detector showed counting rate capabilities in excess of $1 \mathrm{MHz}$, with an energy shift smaller than $1.5 \%$ for a perturbation of approximately $600 \mathrm{keV}$ and a rate of $500 \mathrm{kHz}$. Moreover shifts induced in the measured energy spectrum can be corrected off-line, provided a measurement of the SiPM output current is available. The energy non linearity of the detector was also studied and a value of about $20 \%$ at 10 $\mathrm{MeV}$ for optimized settings was found, which can be corrected for by off-line analysis.

In general, the SiPM based detector we have developed combines compact dimensions, insensitivity to magnetic fields and $\mathrm{MHz}$ counting rate capabilities with only a moderate peak shift. It is therefore suitable for applications to runaway electron studies by gamma-ray bremsstrahlung measurements at $\mathrm{MHz}$ counting rates, particularly as part of multiple line of sight devices, such as gamma-ray cameras.

\section{ACKNOWLEDGMENTS}

This work has been carried out within the framework of the EUROfusion Consortium and has received funding from the Euratom research and training programme 20142018 under grant agreement No 633053. The views and opinions expressed herein do not necessarily reflect those of the European Commission.

\section{REFERENCES}

${ }^{1}$ M. Gobbin et al., Plasma Phys. Control. Fusion 60, 014036 (2017)

${ }^{2}$ G. Pautasso et al., Plasma Phys. Control. Fusion 59, 014046 (2017)

${ }^{3}$ C. Paz-Soldan et al., Phys. Rev. Lett. 118, 255002 (2017)

${ }^{4}$ M. Nocente et al., Nuclear Fusion 55, 123009 (2015)

${ }^{5}$ M. Salewski et al., Nuclear Fusion 55, 093029 (2015)

${ }^{6}$ Ye. O. Kazakov et al., Nature Physics 13, 973-978 (2017)

${ }^{7}$ M. Salewski et al., Nuclear Fusion 57, 056001 (2017)

${ }^{8}$ M Tardocchi et al., Plasma Phys. Control. Fusion 55, 074014 (2013)

${ }^{9}$ A.E. Shevelev et al., Nuclear Fusion 58, 016034 (2018)

${ }^{10} \mathrm{M}$. Nocente et al. "High resolution gamma-ray spectrometer with $\mathrm{MHz}$ capabilities for runaway electron studies at ASDEX Upgrade”, These Proceedings

${ }^{11}$ D.C. Pace et al., Review of Scientific Instruments 87, 043507 (2016)

${ }^{12}$ M. Nocente et al., Nuclear Fusion 57, 076016 (2017)

${ }^{13}$ R.Y. Zhu et al., Journal of Physics: Conference Series 293, 012004 (2011)

${ }^{14} \mathrm{M}$. Nocente et al., Review of Scientific Instruments 85, 11E108 (2014)

${ }^{15}$ D. Rigamonti et al., Journal of Instrumentation 12, C10007 (2017)

${ }^{16}$ D. Rigamonti et al., Review of Scientific Instruments 87, 11E717 (2016)

${ }^{17} \mathrm{M}$. Nocente et al., Review of Scientific Instruments 87, 11E714 (2016)

${ }^{18} \mathrm{M}$. Tardocchi et al., Review of Scientific Instruments 79, 10E524 (2008) 